\title{
Rupture of Arteriovenous Fistula After Transplant Kidney's Biopsy: A Case Report
}

\author{
The Do Ngoc (iD \\ Duc Tran
}

Department of Urology, 108 Military Central Hospital, Hanoi, Vietnam

Correspondence: The Do Ngoc Department of Urology, 108 Military Central Hospital, I Tran Hung Dao Street, Hai Ba Trung District, Hanoi, Vietnam

Tel +84983452468

Email dongocthe@yahoo.com
Background: To comment on the diagnosis and management of a rupture of arteriovenous fistula which is a rare but serious complication after transplant kidney's biopsy.

Case Presentation: The report was performed on a serious case with rupture of arteriovenous fistula after transplant kidney's biopsy in 108 Military Central Hospital. On the 7th day after biopsy, after moving out of the technical room for double J-stent removal, the patient suddenly felt a sharp pain in the transplanted kidney, followed by constant and increasing pain, also complete anuria. We revealed an arteriovenous fistula at the lower pole causing the parenchymal rupture and a large hematoma surround the graft and occupying the retroperitoneum. The emergency operation was done to remove the hematoma and suture the graft's wound containing the arteriovenous fistula to stop the bleeding and eventually salvage the graft.

Conclusion: Rupture of arteriovenous fistula after transplant kidney's biopsy is a rare but severe complication likely lead to graft loss. The patient should be carefully monitored with blood tests and Doppler ultrasound as a routine at least 2 weeks after biopsy.

Keywords: arteriovenous fistula, bleeding, transplant kidney’s biopsy

\section{Introduction}

Renal graft biopsy is indicated after transplantation routinely to collect graft tissue for pathology. ${ }^{1,2}$ It is an invasive procedure so there are many potential complications such as pain, hematuria, subcapsular hematoma, arteriovenous fistula (AVF) ...; may be followed by intensive monitoring, interventional radiology vascular embolization, surgical exploration .... ${ }^{2}$ However, a rupture of AVF causing the compression of the kidney due to a large hematoma a large hematoma is rare in clinical practice. It causes not only blood loss but also graft ischemia leading to graft loss and threatening the patient's survival. We report a case with rupture of arteriovenous fistula on the 7th day after biopsy in the situation of suspected acute rejection which was diagnosed and treated timely.

A 27-year-old Vietnamese woman was admitted to the hospital with end-stage renal disease due to chronic glomerulonephritis in May 2020. She underwent a renal transplant from a living related donor in June 2020; the graft is her mother's left kidney, graft vascular was end-to-side anastomosed to recipient's right external iliac vessels, the ureter was re-implanted into the bladder with modified LichGregoir technique. The graft excreted urine after 5 minutes of reperfusion and the warm ischemia time was about 40 minutes.

Urine volume was 5.5 liters for the first 24 hours after surgery but it decreased to 2.2 liters per 24 hours on average for the next few days. The patient was used 
oral diuretics (furosemide) from the 7 th day after transplant but the serum creatinine was higher than $130 \mathrm{mcmol} /$ L. The acute rejection was supposed so the graft's biopsy was performed with 2 samples. After the procedure, the patient had no fever, no graft pain and there was no decrease in hemoglobin's concentration and red blood cell count.

On the 7 th day after biopsy, cystoscopy was performed to remove the double J-stent. Just after moving out of the technical room, the patient suddenly felt a sharp pain in the transplanted kidney, followed by constant and increasing pain. The patient was given the analgesic and bed rest. Within 1 hour after the onset of pain, the patient could still urinate $100 \mathrm{~mL}$ of clear urine, followed by complete anuria.

At the 4th hour, ultrasound showed that the transplanted kidney in the right iliac fossa was slightly hyperechoic, with $7 \mathrm{~mm}$ perinephric heterogeneous fluid, spreading to the proximal right flank abdominal wall, forming a $74 \times 40 \mathrm{~mm}$ hyperechoic heterogeneous mass, calyces were not dilated and the parenchyma was still well perfused. At the 8th hour, ultrasound showed that there was an $81 \times 48 \mathrm{~mm}$ heterogeneous mass in the right flank, contacted with a $68 \times 60 \mathrm{~mm}$ heterogeneous mass around the transplant kidney; the parenchyma's resistant index (RI) was 0.78 and the arterial RI was 0.86 . The blood test showed a sharp decrease in red blood cell count and hemoglobin (Table 1). The 9th-hour CT-Scan showed that there was an arteriovenous fistula at the lower pole. It caused a parenchymal rupture and large hematoma (Figure 1).

The patient was urgently sent to the hybrid operating room. Unfortunately, the intravascular embolization was not successful after 60 minutes. We decided to operate immediately. The incision was made onto the healed wound. We found that the graft's capsule was peeled off due to a large retroperitoneal hematoma in the right iliac fossa and right flank. After about $500 \mathrm{~mL}$ blood clots were removed, the graft was soft and a $1.5 \mathrm{~cm}$ anterior parenchymal rupture was revealed (Figure 2). We inserted a piece of absorbable hemostats (Surgicel) into the wound and used a 2/0 Catgut chromic to suture it including AVF with 3 simple encircling knots cutting through the graft's parenchyma (Figure 3 ). Then, the graft began to re-excrete the clear urine despite previous anuria.

Postoperatively, the patient had to undergo dialysis 2 times, transfused 3 packs of irradiated red blood cells. The patient was discharged on the 22 nd day after surgery. At the 3rd month and 9th month after discharge, ultrasounds showed that the graft was in normal condition with 0.59 of parenchymal's RI and 0.6 arterial RI; renal pelvis and ureter were not dilated.

\section{Discussion}

Renal graft biopsy has many potential complications. Minor complications were common such as hematuria, arteriovenous fistula, and/or small hematoma. Major complications are usually described as those that require additional treatment, such as transfusion of blood products, surgical or vascular intervention, or prolonged hospitalization, and that are less frequent but can result in significant morbidity. $^{2}$

The overall reported incidence of bleeding in allograft biopsies varies greatly, ranging from $3 \%$ to $16.5 \%$, and the reported incidence of symptomatic AVFs is about $0.4 \% .^{3}$ The management of complications is straightforward. Bed rest itself resolves most of the minor bleeding, but severe bleeding episodes require blood transfusion, radiological intervention, and nephrectomy.

\section{Diagnosis}

Reports showed that this complication occurs mainly in the first week after biopsy. ${ }^{3,4}$ Clinical symptoms of AVF's rupture are often very sudden. The most common first sign is a sharp pain in graft's region. Blood from AVF rupture can cause hematuria, urinary retention or

Table I Results of Blood Test Over Time

\begin{tabular}{|c|c|c|c|c|c|}
\hline Index & $\begin{array}{l}\text { I Day } \\
\text { Before Biopsy }\end{array}$ & $\begin{array}{l}\text { I Day After } \\
\text { Biopsy }\end{array}$ & $\begin{array}{c}5 \text { Day After Biopsy (2 Day } \\
\text { Before AVF's Rupture) }\end{array}$ & $\begin{array}{l}\text { 4th Hour After } \\
\text { AVF's Rupture }\end{array}$ & $\begin{array}{l}\text { 8th Hour After } \\
\text { AVF's Rupture }\end{array}$ \\
\hline Red blood cells (T/L) & 4.25 & 4.36 & 4.47 & 4.01 & 3.47 \\
\hline Hemoglobin $(g / L)$ & 93 & 95 & 98 & 87 & 76 \\
\hline Hematocrit (L/L) & 0.31 & 0.31 & 0.32 & 0.28 & 0.25 \\
\hline Urea $(\mathrm{mmol} / \mathrm{L})$ & 16.1 & 19.7 & 16,6 & 20.3 & - \\
\hline Creatinine $(\mu \mathrm{mol} / \mathrm{L})$ & 229 & 235 & 240 & 302 & - \\
\hline
\end{tabular}




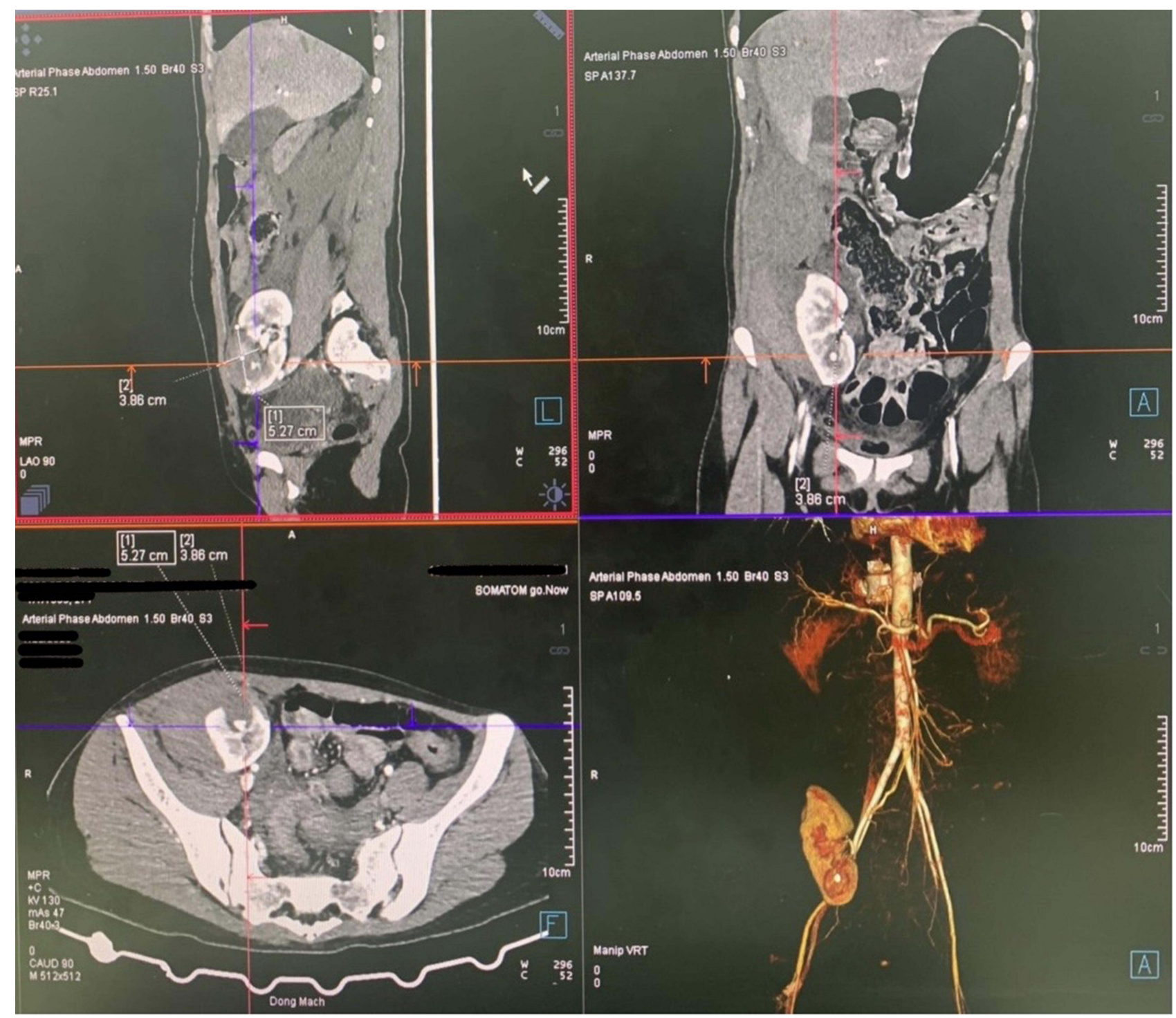

Figure I The 9th-hour CT-Scan showed that there was an arteriovenous fistula at the lower pole. It caused the parenchymal rupture and a large hematoma.

renal colic due to blood clots, or forming subcapsular hematoma, as well as a large retroperitoneal hematoma in severe cases like our case in this report.

Also, we revealed that the compression of the kidney due to the large hematoma, which is a recognized cause of graft ischemia. So that, with the anemia due to blood loss, the amount of urine gradually decreases with increasing serum creatinine. Patients quickly become anuria. ${ }^{3,4}$

\section{Management}

First, we performed vascular embolization to stop bleeding but it was not successful. So that we had to do a surgical exploration to remove the hematoma and suture the AVF. We consider that, even if the intra- vascular intervention was successful, still had to have open surgery to decompress the kidney. The evidence was that the patient was completely anuria, and immediately after decompression, urine excretion returned.

Other reports also had the same management, the graft's function improved just after removing hematoma with the increase in urine output and the decrease in serum creatinine. $^{2,4}$

\section{Prognostics and Prevention}

Redfield (2015) recommends close monitoring of patients during the first week after biopsy; the factors predicting the risk of complications are a decrease in hematocrit/hemoglobin and an increase in serum urea/ 


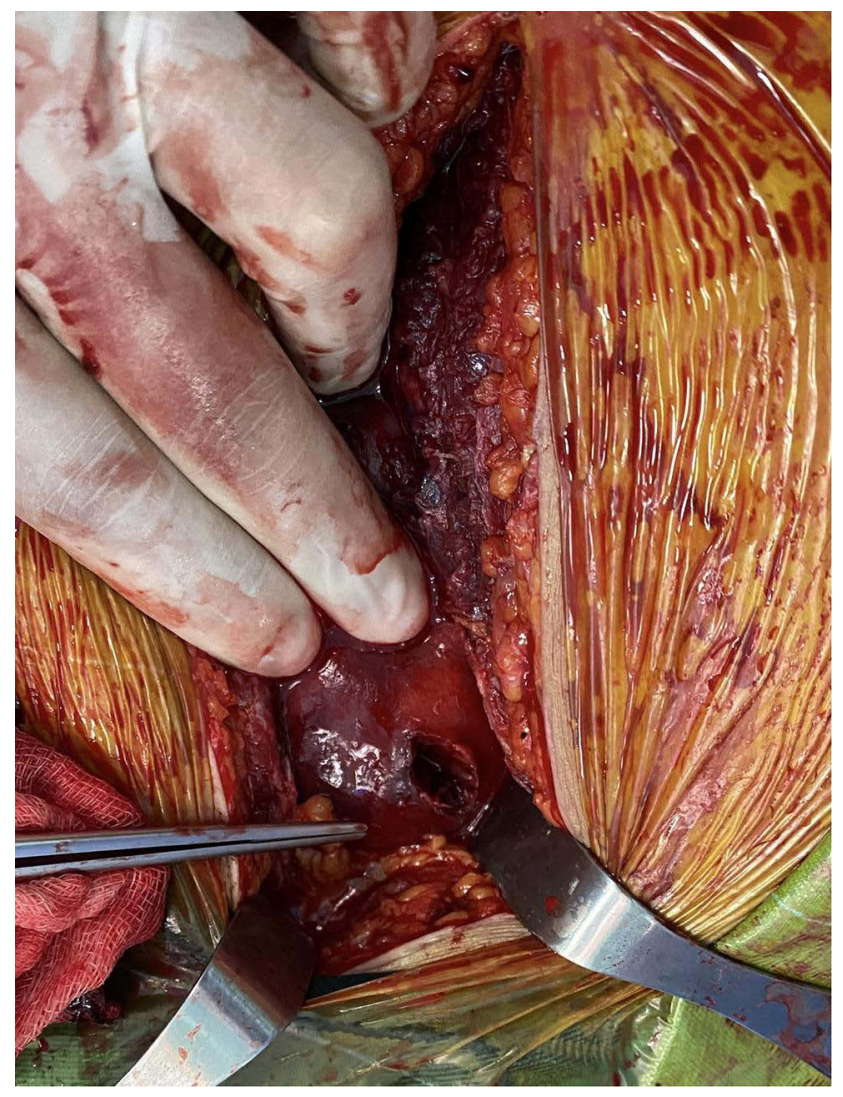

Figure 2 The wound was at the anterior lower pole.

creatinine. $^{5}$ However, Morgan (2019) performed a Doppler ultrasound of the transplanted kidney 10 min immediately after the biopsy procedure and then performed routine follow-up. ${ }^{2}$

In our case, there was no change in the blood test before the accident and we did not use any imaging examination. If the patient had been monitored by ultrasound and managed the arteriovenous fistula with embolization, perhaps that complication did not happen.

Fang (2019) recommends using CDFI (Colour Doppler Flow Imaging) mode while determining the puncture site. ${ }^{4}$ The site for biopsy of the transplanted kidney in the cortical margin not only increases the success rate to over $95 \%$ but also reduces the risk of bleeding to less than $1 \%$. $^{5,6}$

\section{Conclusion}

We have found that rupture of AVF after biopsy of transplant kidney can cause severe blood loss, subcapsular or retroperitoneal hematoma, results in graft ischemia. A large hematoma should be removed by an emergency operation. Within 2 weeks after biopsy, it is necessary to

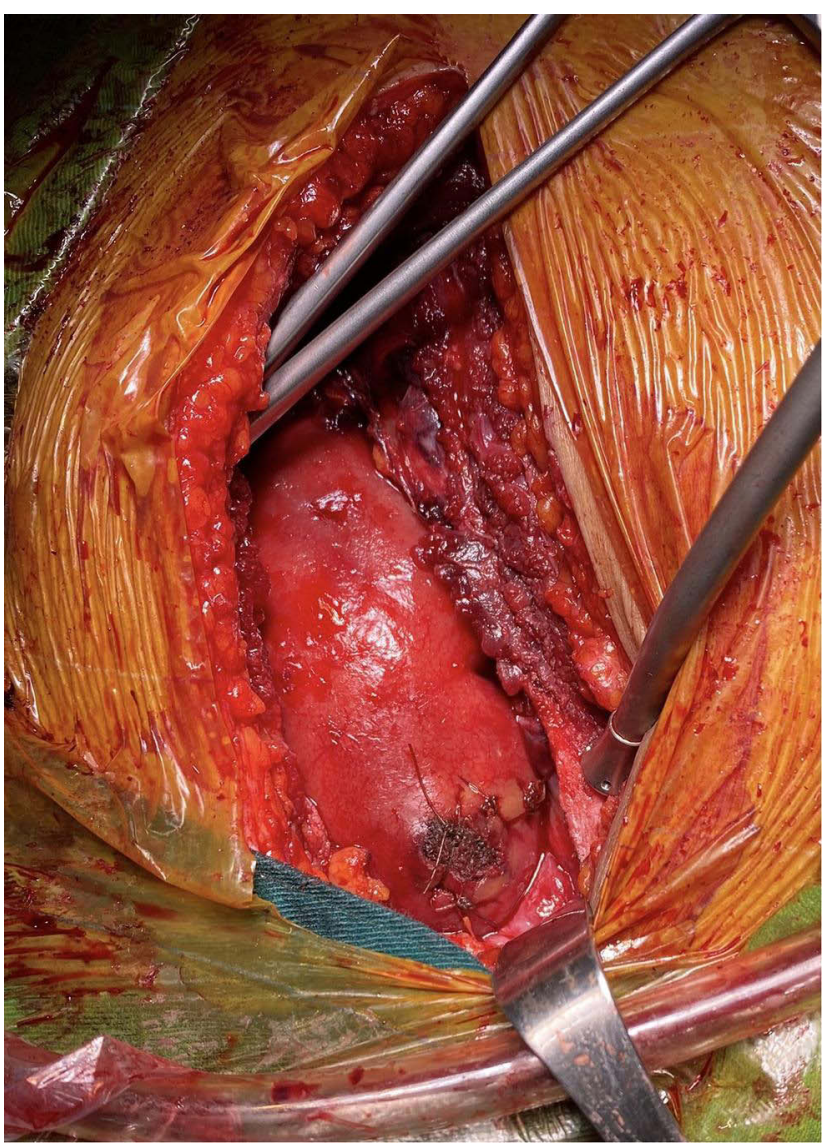

Figure 3 The wound was sutured.

closely monitor patients with blood tests and Doppler ultrasound as a routine.

\section{Abbreviations}

AVF, arteriovenous fistula; RI, resistant index.

\section{Ethics Approval and Consent to Participate}

The patient has received the kidney from her mother. The donation was voluntary with written informed consent, and conducted in accordance with the Declaration of Istanbul. The case reports received approval for publication from the Ethics Committee of 108 Military Central Hospital.

\section{Consent for Publication}

Written informed consent for publication of the clinical details and clinical images was obtained from the patient.

\section{Disclosure}

The authors declare that they have no conflicts of interest in this work. 


\section{References}

1. Vietnam Society of Organ Transplatation. Monitoring and treatment after kidney transplant. VSOT guideline of kidney transplant. Hanoi: Medical Publishing House; 2017:92-241.

2. Morgan TA, Chandran S, Burger IM, et al. Complications of ultrasound-guided renal transplant biopsies. Am $J$ Transplant. 2016;16(4):1298-1305. doi:10.1111/ajt.13622

3. Prasanna A, Weerakkody RM, Wijewickrama ES, et al. Salvage of bleeding renal allograft following biopsy, with suture technique: a case report. J Med Case Rep. 2016;10(1):1-6. doi:10.1186/s13256-016-0870-2
4. Fang J, Li G, Xu L, et al. Complications and clinical management of ultrasound-guided renal allograft biopsies. Transl Androl Urol. 2019;8(4):292-296. doi:10.21037/tau.2019.07.23

5. Redfield RR, McCune KR, Rao A, et al. Nature, timing, and severity of complications from ultrasound-guided percutaneous renal transplant biopsy. Transpl Int. 2016;29(2):167-172. doi:10. $1111 /$ tri. 12660

6. Patel MD, Phillips CJ, Young SW, et al. US-guided renal transplant biopsy: efficacy of a cortical tangential approach. Radiology. 2010;256(1):290-296. doi:10.1148/radiol.10091793

\section{Publish your work in this journal}

Research and Reports in Urology is an international, peer-reviewed, open access journal publishing original research, reports, editorials, reviews and commentaries on all aspects of adult and pediatric urology in the clinic and laboratory including the following topics: Pathology, pathophysiology of urological disease; Investigation and treatment of urological disease; Pharmacology of drugs used for the treatment of urological disease. The manuscript management system is completely online and includes a very quick and fair peer-review system, which is all easy to use. Visit http://www.dovepress.com/ testimonials.php to read real quotes from published authors. 